\title{
An L-Shaped Relationship Between Serum Iron and Stroke-Associated Pneumonia
}

This article was published in the following Dove Press journal:

Clinical Interventions in Aging

Jia $\mathrm{Li}^{1}$

Liang Feng'

Qiqi Huang ${ }^{2}$

Wenwei Ren'

'Department of Neurology, The First Affiliated Hospital of Wenzhou Medical University, Wenzhou, 325000, People's Republic of China; ${ }^{2}$ Department of Cardiac Care Unit, The First Affiliated Hospital of Wenzhou Medical University, Wenzhou, 325000, People's Republic of China
Correspondence: Wenwei Ren Department of Neurology, The First Affiliated Hospital of Wenzhou Medical University, Wenzhou, 325000, Zhejiang

Province, People's Republic of China

Tel/Fax +8657755554543

Email rww037@I26.com

Qiqi Huang

Department of Cardiac Care Unit, The First Affiliated Hospital of Wenzhou

Medical University, Wenzhou, 325000,

Zhejiang Province, People's Republic of China

Tel/Fax +8657755554543

Email wyyyhqq@I26.com
Objective: Pneumonia is a common complication in patients with stroke. There was a close relationship between serum iron and inflammatory response. This study aimed to explore the relationship between serum iron levels and stroke-associated pneumonia (SAP).

Methods: Patients with acute stroke were recruited from the First Affiliated Hospital of Wenzhou Medical University and divided into SAP group and Non-SAP group. The demographic and clinical data of the patients were collected via the medical records, and the blood samples were collected within 24 hours after admission. The predictive value of serum iron to SAP was evaluated by receiver operating characteristic curve (ROC) and binary Logistic regression models. A restricted cubic spline (RCS) was used to furtherly clarify the relationship between serum iron and the risk of SAP.

Results: A total of 906 participants were enrolled, including Non-SAP group $(n=755)$ and SAP group $(n=151)$. Serum iron levels in the SAP group were significantly lower than those in the Non-SAP group $(9.77 \pm 5.61$ vs $14.01 \pm 6.80, \mathrm{P}<0.001)$. Logistic regression showed that patients with high serum iron levels $(\geq 7.8 \mu \mathrm{mol} / \mathrm{L})$ showed a lower risk of SAP (OR $=0.43$, 95\% CI, 0.27-0.69, P $<0.001)$. Besides, the RCS model showed that there was an L-shaped relationship between the serum iron and risk of SAP (P for non-linearity: 0.014).

Conclusion: Low serum iron level was a risk factor for SAP, and there was an L-shaped relationship between them. Stroke patients with low serum iron levels should be alert to the risk of SAP.

Keywords: stroke, pneumonia, serum iron

\section{Introduction}

Stroke is one of the main causes of human death, and its prognosis depends largely on complications such as pneumonia. ${ }^{1}$ Studies have shown that about one-third of early death and poor prognosis after stroke are caused by potential preventable stroke-related complications, especially infection. ${ }^{2}$ However, the prediction of complications is still challenging at present. $^{3}$

Iron is an essential element for humans, it is necessary for many metabolic processes such as oxygen transport, electron transport, and DNA synthesis. ${ }^{4}$ Besides, iron is also involved in human redox reactions, producing reactive hydroxyl radicals, and damaging macromolecules such as DNA and proteins. ${ }^{5}$ Serum iron is a medical laboratory test that measures the amount of circulating iron. Previous studies had demonstrated that inflammatory factors could affect iron metabolism and reduce serum iron levels. ${ }^{6-9}$ In the etiological study of anemia in the elderly, it was also found that about two-thirds of cases had iron deficiency and chronic inflammation. $^{10,11}$ 
Stroke-associated pneumonia (SAP) is one of the most common complications after stroke, with an incidence of $11.3-31.3 \%{ }^{12}$ SAP may influence the prognosis of stroke, including prolonged hospitalization, decreased cognitive function, depression, and increased disability and mortality. ${ }^{13,14}$ A previous cross-sectional study including 101 stroke patients revealed that patients with SAP showed lower serum iron levels than those without SAP, but this study did not furtherly analyze whether there was a linear relationship or not between serum iron and the risk of SAP, and the sample size was relatively small. ${ }^{15}$ Our study will furtherly explore the relationship between serum iron and SAP with larger sample size.

\section{Methods}

\section{Subjects}

This was a cross-sectional study, and patients with acute stroke including cerebral infarction and cerebral hemorrhage were recruited from the First Affiliated Hospital of Wenzhou Medical University between 2016.11 and 2020.04. The inclusion criteria were as follows: (1) age $\geq 18$ years; (2) stroke onset within one week; (1) acute stroke confirmed by MRI or CT. Exclusion criteria: (1) active infection or fever within 2 weeks before admission; (2) patients treated with iron supplementation; (3) preexisting dysphagia; (4) pre-stroke pneumonia or communityacquired pneumonia; (5) other severe diseases including tumors, blood system disease, liver or kidney dysfunction; (6) medical records incomplete.

A total of 1243 patients were screened, 337 patients were excluded, and 906 patients were finally included in this study, of which 151 patients were diagnosed as SAP and 755 with Non-SAP (Figure 1). SAP was diagnosed by two experienced neurologists according to the modified Centers for Disease Control and Prevention criteria of hospital-acquired pneumonia, ${ }^{16}$ combining the clinical and laboratory indicators of respiratory infection, and was confirmed by chest X-ray and computed tomography. ${ }^{17}$

The study was approved by the Ethics Committee of the First Affiliated Hospital of Wenzhou Medical University, and was complied with the Declaration of Helsinki. As the study was retrospective and all the patient data were anonymous, the patient informed consent was waived.

\section{Data Collection}

Demographic and clinical data were collected from the medical record system. Blood samples were collected from patients the next morning after overnight fasting within 24 hours of admission before the diagnosis of SAP. Laboratory data included serum iron, serum ferritin, glomerular filtration rate (GFR), red blood cell, hemoglobin, leukocyte, and platelet count. The normal range of serum iron in this study is $12.5 \sim 32.2 \mu \mathrm{mol} / \mathrm{L}$. The swallowing function was evaluated and determined within

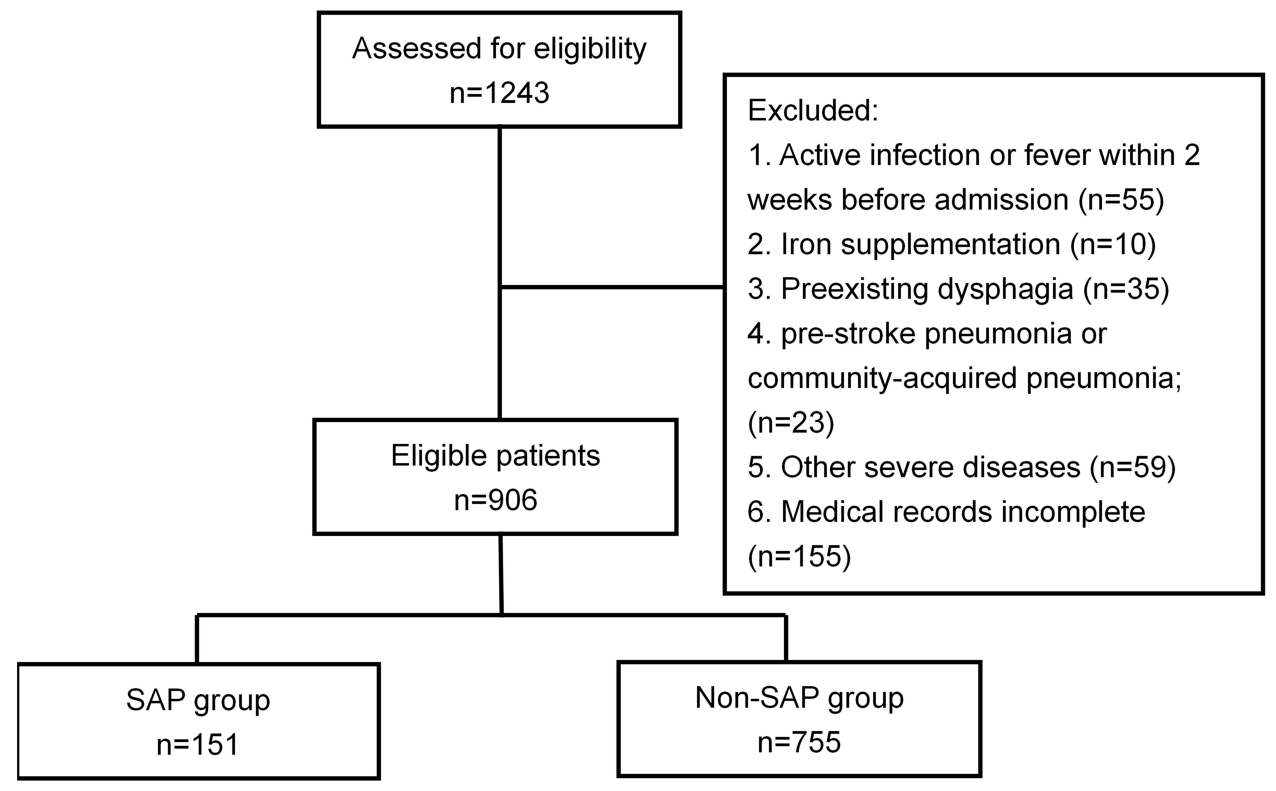

Figure I Flow chart of the study.

Abbreviation: SAP, stroke-associated pneumonia. 
24hrs after admission by trained specialists using V-VST (Volume viscosity screening Test). ${ }^{18}$ After the V-VST test, the diet was determined by the specialists, which mainly included four types: general diet, semi-liquid diet, paste meal, and nasogastric tube feeding. In this study, the diet was used as an index of the swallowing function. Stroke severity after admission was assessed by the National Institutes of Health Stroke Score (NIHSS).

\section{Statistical Analysis}

Classified data were expressed as percentages and analyzed by the chi-square test or Fisher exact test. Continuous variables were presented as the mean \pm standard deviation or median and interquartile range (IQR) based on the distribution of data and analyzed by $t$-test or the Mann-Whitney U-test. Receiver operating characteristic (ROC) curve analysis was adopted to determine the best cutoff values for serum iron, ferritin, and leukocytes. Serum iron was furtherly stratified according to the cutoff value. Binary Logistic regression model was adopted to clarify the relationship between serum iron and SAP, with the confounding factors being controlled. Besides, we also used restricted cubic splines with four knots at the 5th, 35th, 65th, and 95th centiles to flexibly model the association between serum iron and SAP risk.

All statistical tests were two-sided, and values of $\mathrm{P}<$ 0.05 were considered statistically significant. All data were analyzed using SPSS21.0 and R (version 3.6.3).

\section{Results}

\section{Characteristics Between SAP and Non-SAP Group}

As shown in Table 1, serum iron levels in the SAP group were significantly lower than the Non-SAP group $(9.77$ \pm 5.61 vs. $14.01 \pm 6.80, \mathrm{P}<0.001)$. Besides, there were significant differences in age, stroke type (cerebral infarction, cerebral hemorrhage), swallowing function, atrial fibrillation, NIHSS score, ferritin, leukocytes, red blood cell count, hemoglobin, and glomerular filtration rate between the two groups (all $\mathrm{P}<0.05$ ). There were no differences in the time intervals (day) from the time point of blood collection to the diagnosis of SAP between these two groups $(2.00(1.00,4.00)$ vs $2.00(1.00,4.00), \mathrm{P}=0.607)$.

\section{Serum Iron and SAP}

According to the normal range of serum iron levels in our laboratory $(12.5 \sim 32.2 \mu \mathrm{mol} / \mathrm{L}), 50.9 \%$ (461) of the patients were deficient in iron, $47.7 \%$ (432) in the normal range, and $1.4 \%(13)$ in the high range. Given the small number of patients in the high range, patients in the high range were combined into the normal range. Analyzed by chi-square test, the results showed that the percentage of SAP in the iron deficiency group was significantly higher than that in the normal group $\left(23.9 \%\right.$ vs. $9.2 \%, \chi^{2}=34.98$, $\mathrm{P}<0.001$ ).

As was shown in Figure 2, the AUC (area under the curve) of serum iron (AUC $=0.70,95 \% \mathrm{CI}, 0.67-0.73$ ) was significantly higher than ferritin $(\mathrm{AUC}=0.57,95 \% \mathrm{CI}$, 0.$54-0.60, \mathrm{P}<0.001)$. The AUC of leukocytes $(\mathrm{AUC}=0.67$, 95\% CI, 0.64-0.70) showed no difference to serum iron and ferritin (all $\mathrm{P}>0.05$ ). The ROC curve of serum iron exhibited $84.24 \%$ specificity and $46.36 \%$ sensitivity, with the best cutoff point of $7.8 \mu \mathrm{mol} / \mathrm{L}$. Then, the serum iron was stratified according to the cutoff point and furtherly analyzed by binary Logistic regression analysis. The result was showed by the forest plot. As was shown in the forest plot (Figure 3), patients with serum iron $\geq 7.8 \mu \mathrm{mol} / \mathrm{L}$ had a significantly lower risk of SAP (OR $=0.43,95 \% \mathrm{CI}$, $0.27-0.69, \mathrm{P}<0.001)$, with the confounding factors being controlled.

In Figure 4, we used restricted cubic splines to flexibly model and visualize the relationship between serum iron and the risk of SAP. The figure showed that when the serum iron was at the lowest level, the risk of SAP was the highest. With the increase of serum iron levels, the risk of SAP decreased rapidly. However, when the serum iron level reached around $12.5 \mu \mathrm{mol} / \mathrm{L}$, the risk of SAP got relatively flat (P for non-linearity 0.014 ). As was shown in Table 2, serum iron levels were furtherly stratified into quantiles $(\leq 8.60,8.61-12.30,12.31-16.80, \geq 16.81 \mu \mathrm{mol} / \mathrm{L})$ in the logistic regression model, with the last quantile being the reference. We found the results remained unchanged, lower serum iron levels $(\leq 8.60 \mu \mathrm{mol} / \mathrm{L})$ were associated with a higher risk of SAP $(\mathrm{OR}=2.24,95 \% \mathrm{CI}$, $1.15-4.36, \mathrm{P}=0.018$ ).

\section{Other Risk Factors of SAP}

As shown in Figure 3, stroke type, age, swallowing function, NIHSS score, serum ferritin, and leukocyte were all risk factors for SAP $(\mathrm{P}<0.05)$.

\section{Discussion}

There were two main findings of this study. Firstly, lower serum iron levels were associated with a higher risk of SAP. Secondly, there was an L-shaped relationship between serum iron and the risk of SAP. To our 
Table I Comparison of Characteristics Between Non-SAP and SAP Group

\begin{tabular}{|c|c|c|c|c|c|}
\hline Variables & Total $(n=906)$ & Non-SAP $(n=755)$ & $\operatorname{SAP}(n=151)$ & $\chi^{2} / t / U$ & $P$ \\
\hline Age, Mean \pm SD & $67.23 \pm 12.36$ & $66.47 \pm 12.24$ & $71.07 \pm 12.26$ & -4.209 & $<0.001$ \\
\hline Gender (male), n (\%) & $548(60.49)$ & $456(60.40)$ & $92(60.93)$ & 0.001 & 0.976 \\
\hline BMI, Mean \pm SD & $23.65 \pm 2.69$ & $23.72 \pm 2.60$ & $23.33 \pm 3.06$ & 1.445 & 0.150 \\
\hline Hypertension, n (\%) & $614(67.77)$ & $514(68.08)$ & $100(66.23)$ & 0.122 & 0.727 \\
\hline Heart disease, n (\%) & $102(11.26)$ & $84(11.13)$ & $18(11.92)$ & 0.02 & 0.888 \\
\hline Diabetes, n (\%) & $\mid 84(20.3 \mid)$ & 157 (20.79) & $27(17.88)$ & 0.492 & 0.483 \\
\hline Kidney disease, n (\%) & $16(1.77)$ & II (I.46) & $5(3.3 I)$ & Fisher & 0.164 \\
\hline Tuberculosis, n (\%) & $7(0.77)$ & $5(0.66)$ & $2(1.32)$ & Fisher & 0.330 \\
\hline Liver disease, n (\%) & $16(1.77)$ & $14(1.85)$ & $2(1.32)$ & Fisher & I \\
\hline Atrial fibrillation, n (\%) & $137(15.12)$ & $101(13.38)$ & $36(23.84)$ & 9.934 & 0.002 \\
\hline Smoking, n (\%) & $305(33.66)$ & $25 I(33.25)$ & $54(35.76)$ & 0.253 & 0.615 \\
\hline Drinking, n (\%) & 279 (30.79) & $235(31.13)$ & $44(29.14)$ & 0.149 & 0.699 \\
\hline Stroke type (Hemorrhage), n (\%) & $375(4 \mid .39)$ & $290(38.4 I)$ & $85(56.29)$ & 15.856 & $<0.001$ \\
\hline Swallowing function, $\mathrm{n}$ (\%) & & & & 88.76 & $<0.001$ \\
\hline General diet & $159(17.55)$ & $157(20.79)$ & $2(1.32)$ & & \\
\hline Semi-liquid diet & $572(63.13)$ & $489(64.77)$ & $83(54.97)$ & & \\
\hline Paste meal & $31(3.42)$ & $23(3.05)$ & $8(5.30)$ & & \\
\hline Nasal feeding & I44 (I5.89) & $86(11.39)$ & $58(38.4 I)$ & & \\
\hline NIHSS, Median (IQR) & $5.00(2.00,10.00)$ & $4.00(2.00,9.00)$ & $10.00(4.50,15.00)$ & $33,192.5$ & $<0.001$ \\
\hline Onset time (days), Median (IQR) & $1.00(1.00,3.00)$ & $1.00(1.00,3.00)$ & $1.00(1.00,3.00)$ & $24,013.0$ & 0.262 \\
\hline Serum iron $(\mu \mathrm{mol} / \mathrm{L})$, Mean $\pm S D$ & $|3.3| \pm 6.80$ & $|4.0| \pm 6.80$ & $9.77 \pm 5.61$ & 8.166 & $<0.001$ \\
\hline Serum ferritin $(\mu g / L)$, Mean $\pm S D$ & $227.14 \pm 238.88$ & $214.01 \pm 201.20$ & $292.75 \pm 368.22$ & -2.552 & 0.012 \\
\hline Leukocyte count (I0^9/L), Mean \pm SD & $7.99 \pm 2.74$ & $7.67 \pm 2.39$ & $9.58 \pm 3.65$ & -6.174 & $<0.001$ \\
\hline Red blood cell $\left(10^{\wedge} \mid 2 / L\right)$, Mean $\pm S D$ & $4.42 \pm 0.53$ & $4.45 \pm 0.52$ & $4.31 \pm 0.57$ & 2.697 & 0.008 \\
\hline Hemoglobin (g/L), Mean \pm SD & $135.89 \pm 16.67$ & $136.57 \pm 16.36$ & $132.50 \pm 17.8 \mid$ & 2.595 & 0.010 \\
\hline Platelet count $\left(10^{\wedge} 9 / \mathrm{L}\right)$, Mean \pm SD & $214.49 \pm 66.50$ & $215.63 \pm 66.43$ & $208.78 \pm 66.78$ & 1.152 & 0.251 \\
\hline GFR, Mean \pm SD & $88.60 \pm 20.45$ & $89.99 \pm 19.43$ & $81.64 \pm 23.82$ & 4.047 & $<0.001$ \\
\hline
\end{tabular}

Abbreviations: SAP, stroke-associated pneumonia; BMI, body mass index; NIHSS, National Institutes of Health Stroke Scale; GFR, glomerular filtration rate; IQR, interquartile range; SD, standard deviation; Fisher, Fisher exact test.

knowledge, this is the first study to find a non-linear relationship between serum iron and the risk of SAP.

In this study, we found that up to $50.9 \%$ of stroke patients were in a state of iron deficiency, and the percentage of SAP in these patients was significantly higher than that in normal patients $(23.9 \%$ vs $9.2 \%)$. Further analysis found that patients with serum iron levels $\geq 7.8 \mu \mathrm{mol} / \mathrm{L}$ had a significantly lower risk of SAP and the RCS model showed an L-shaped relationship between serum iron and SAP. The relationship between inflammation and iron metabolism was still not very clear. Previous studies had found that many microbes were highly dependent on an adequate supply of iron and absorbed iron from the host through various pathways. ${ }^{19}$ Therefore, the consumption 


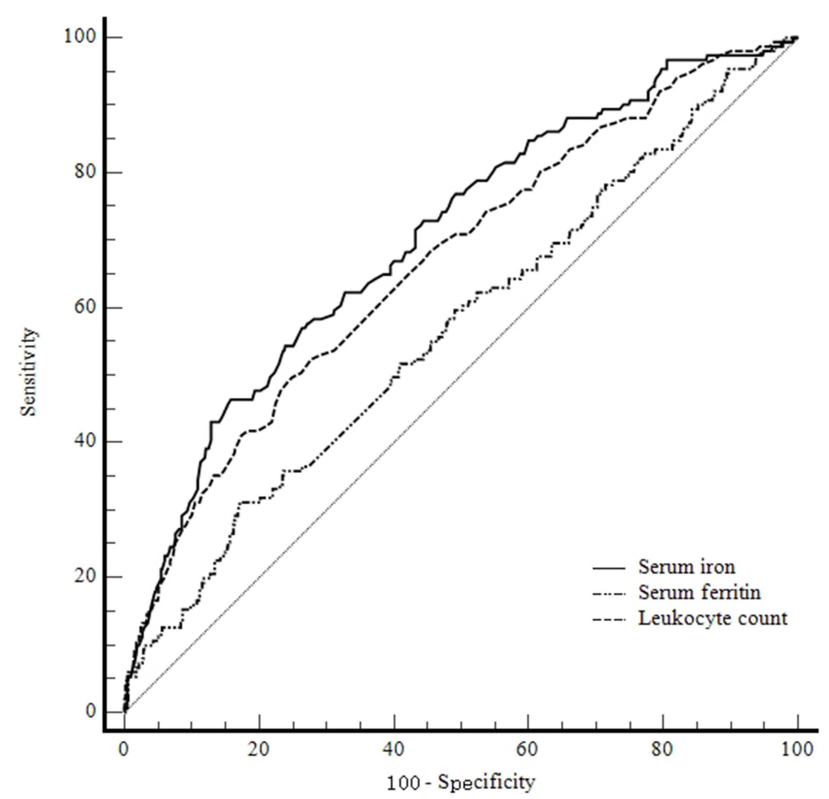

Figure 2 Diagnostic value of serum iron, serum ferritin and leukocyte count for SAP assessed via ROC curve analysis.

Abbreviations: SAP, stroke-associated pneumonia; ROC, receiver operating characteristic.

of iron by microbes might be one of the reasons for the decrease of serum iron levels in patients with SAP. Besides, macrophages played an important role in iron recycling. Iron recycling by macrophages accounted for about $95 \%$ of the daily needs under physiological conditions. ${ }^{20}$ Accumulating studies had found that inflammation could impair macrophage iron metabolism and iron homeostasis, which resulted in low circulating iron levels and high ferritin levels. ${ }^{20}$ It was worth noting that the present study also observed low serum iron levels and high ferritin levels in patients with SAP, which was consistent with previous findings.

The L-shaped relationship between serum iron and the risk of SAP indicated that patients with iron deficiency $(<12.5 \mu \mathrm{mol} / \mathrm{L})$ had a higher risk of SAP and a small change of serum iron might have a great impact on the risk of SAP. However, the risk of SAP was low and stable for the patients in the normal range of serum iron levels $(12.5 \sim 32.2 \mu \mathrm{mol} / \mathrm{L})$. Because few patients had high serum iron levels $(>32.2 \mu \mathrm{mol} / \mathrm{L})$, we were unable to know the changing trend of the risk of SAP in patients with high serum iron levels. While, it was supposed that supplying iron to microbes could increase their pathogenicity. ${ }^{19}$ For example, studies had observed that iron overload could reduce the survival rate of AIDS patients with pneumocystis carinii pneumonia, ${ }^{21}$ and iron supplementation could increase the risk of malaria. ${ }^{22}$ Given above, we supposed that there might be a U-shaped relationship between serum iron and the risk of SAP. Future studies were needed to furtherly explore this issue. In addition, this study also found that age, swallowing function, NIHSS score, and stroke type were all associated with SAP, which were consistent with previous studies. ${ }^{3,23}$

There were several limitations in this study. Firstly, this study was a cross-sectional study, which could not draw a causal relationship between serum iron and SAP; Secondly, the SAP and Non-SAP group did not match very well, which might bring bias to the results. Therefore, we adopted logistic regression models to control the confounding factors; Thirdly, the lack of diet habit information

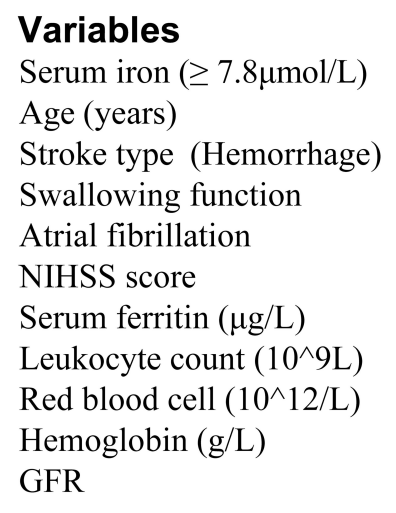

Variables

Serum iron $(\geq 7.8 \mu \mathrm{mol} / \mathrm{L})$

Stroke type (Hemorrhage)

Atrial fibrillation

NIHSS score

Serum ferritin $(\mu \mathrm{g} / \mathrm{L})$

Leukocyte count $\left(10^{\wedge} 9 \mathrm{~L}\right)$

Hemoglobin $(\mathrm{g} / \mathrm{L})$

GFR

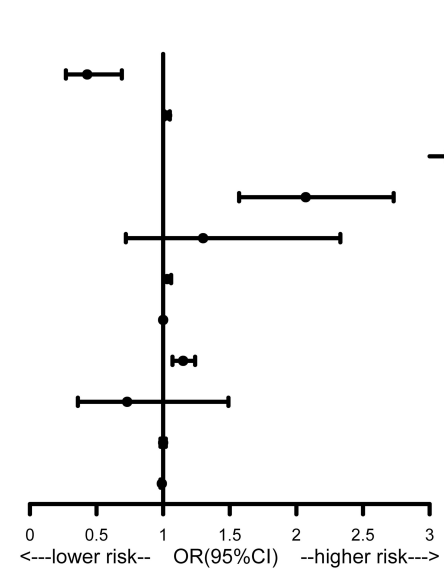

Figure 3 Forest plot of odds ratios for SAP.

Abbreviations: NIHSS, National Institutes of Health Stroke Scale; 95\% CI, 95\% confidence interval; SAP, stroke-associated pneumonia; GFR, glomerular filtration rate; OR, odds ratio. 


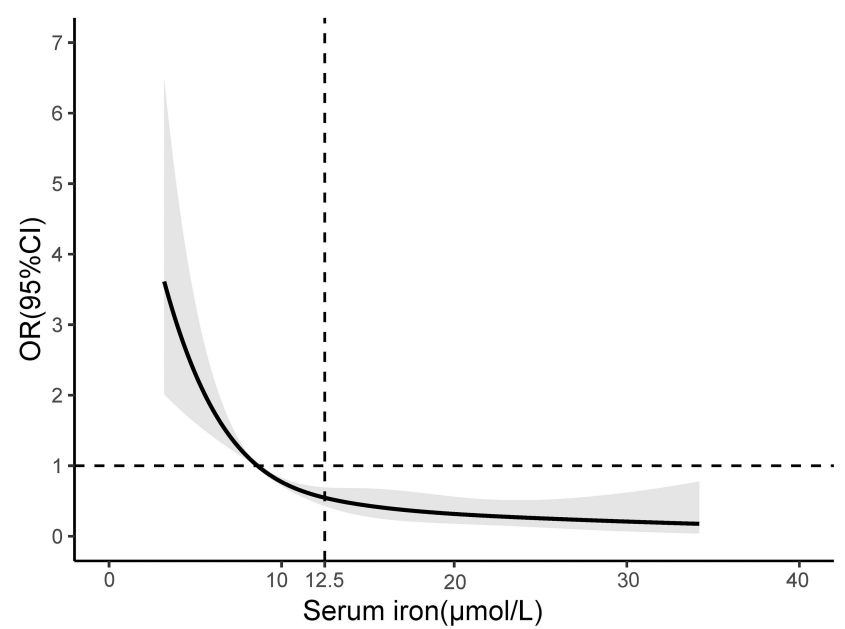

Figure 4 Association between serum iron levels and risk of SAP using restricted cubic spline analysis.

Abbreviations: SAP, stroke-associated pneumonia; OR, odds ratio; $95 \% \mathrm{Cl}, 95 \%$ confidence interval.

including dietary alcohol consumption might have an influence on the results, which should be taken into consideration when screening the patients in future studies; Fourthly, serum iron levels might have daily fluctuations. Therefore, interpretation of the results needed more caution.

Table 2 Logistic Model of the Clinical Determinants of SAP

\begin{tabular}{|c|c|c|}
\hline Variables & OR $(95 \% \mathrm{Cl})$ & $P$ value \\
\hline Serum iron $(\leq 8.60 \mu \mathrm{mol} / \mathrm{L})$ & $2.24(1.15-4.36)$ & 0.018 \\
\hline Serum iron $(8.6 \mathrm{I}-12.30 \mu \mathrm{mol} / \mathrm{L})$ & $1.64(0.83-3.26)$ & 0.155 \\
\hline Serum iron $(|2.31-| 6.80 \mu \mathrm{mol} / \mathrm{L})$ & $1.25(0.61-2.55)$ & 0.545 \\
\hline Serum iron $(\geq 16.81 \mu \mathrm{mol} / \mathrm{L})$ & Reference & \\
\hline Age (years) & $1.03(1.01-1.05)$ & 0.003 \\
\hline Stroke type (Hemorrhage) & $5.14(3.07-8.62)$ & $<0.001$ \\
\hline Swallowing function & $2.10(1.60-2.77)$ & $<0.001$ \\
\hline Atrial fibrillation & $1.26(0.70-2.25)$ & 0.445 \\
\hline NIHSS score & $1.03(1.01-1.06)$ & 0.022 \\
\hline Serum ferritin $(\mu g / L)$ & $1.00(1.00-1.00)$ & 0.025 \\
\hline Leukocyte count (10^9L) & $1.16(1.08-1.25)$ & $<0.001$ \\
\hline Red blood cell $\left(10^{\wedge} \mid 2 / L\right)$ & $0.7 \mid(0.35-1.45)$ & 0.351 \\
\hline Hemoglobin $(g / L)$ & $1.00(0.98-1.02)$ & 0.988 \\
\hline GFR & $0.99(0.98-1.00)$ & 0.109 \\
\hline
\end{tabular}

Abbreviations: NIHSS, National Institutes of Health Stroke Scale; SAP, strokeassociated pneumonia; GFR, glomerular filtration rate; OR, odds ratio; $95 \% \mathrm{Cl}, 95 \%$ confidence interval.

\section{Conclusion}

In conclusion, lower serum iron levels were associated with a higher risk of SAP, and there was an L-shaped relationship between them. Clinically, stroke patients with low serum iron levels should be alert to the risk of SAP, and necessary examinations were needed to achieve early detection and treatment. Future studies were needed to furtherly explore the relationship between the iron supplement and outcomes of SAP.

\section{Abbreviations}

SAP, stroke-associated pneumonia; ROC, area under the receiver operating characteristic curve; AUC, area under the curve; RCS, restricted cubic spline; CI, confidence interval; GFR, glomerular filtration rate; V-VST, Volume viscosity screening Test; NIHSS, National Institutes of Health Stroke Score. IQR, interquartile range; BMI, Body Mass Index; Fisher, Fisher exact test.

\section{Data Sharing Statement}

The data supporting this study are available from the corresponding author Wenwei Ren for reasonable request.

\section{Acknowledgments}

The authors thank the patients of this study for their important contributions. This work was supported by Municipal Sci-Tech Bureau Programs (no. Y2020427 and Y20170335).

\section{Author Contributions}

All authors made substantial contributions to conception and design, acquisition of data, or analysis and interpretation of data; took part in drafting the article or revising it critically for important intellectual content; agreed to submit to the current journal; gave final approval of the version to be published; and agree to be accountable for all aspects of the work.

\section{Disclosure}

The authors have no conflicts of interest to disclose.

\section{References}

1. Kumar S, Selim MH, Caplan LR. Medical complications after stroke. Lancet Neurol. 2010;9(1):105-118. doi:10.1016/S1474-4422(09) 70266-2

2. Koennecke HC, Belz W, Berfelde D, et al. Factors influencing in-hospital mortality and morbidity in patients treated on a stroke unit. Neurology. 2011;77(10):965-972. doi:10.1212/WNL.0b $013 \mathrm{e} 31822 \mathrm{dc} 795$ 
3. Hoffmann S, Malzahn U, Harms H, et al. Development of a clinical score (A2DS2) to predict pneumonia in acute ischemic stroke. Stroke. 2012;43(10):2617-2623. doi:10.1161/STROKEAHA.112.653055

4. Gutierrez-Bedmar M, Olmedo P, Gil F, et al. Low serum iron levels and risk of cardiovascular disease in high risk elderly population: nested case-control study in the PREvencion con DIeta MEDiterranea (PREDIMED) trial. Clin Nutr. 2020.

5. Wardman P, Candeias LP. Fenton chemistry: an introduction. Radiat Res. 1996;145(5):523-531. doi:10.2307/3579270

6. Ferrucci L, Semba RD, Guralnik JM, et al. Proinflammatory state, hepcidin, and anemia in older persons. Blood. 2010;115 (18):3810-3816. doi:10.1182/blood-2009-02-201087

7. Kervinen H, Tenkanen L, Palosuo T, et al. Serum iron, infection and inflammation; effects on coronary risk. Scand Cardiovasc J. 2004;38 (6):345-348. doi:10.1080/14017430410011003

8. Roy CN, Andrews NC. Anemia of inflammation: the hepcidin link. Curr Opin Hematol. 2005;12(2):107-111. doi:10.1097/00062752200503000-00001

9. Rogers JT. Ferritin translation by interleukin-1and interleukin-6: the role of sequences upstream of the start codons of the heavy and light subunit genes. Blood. 1996;87(6):2525-2537. doi:10.1182/blood. V87.6.2525.bloodjournal8762525

10. Joosten E, Pelemans W, Hiele M, et al. Prevalence and causes of anaemia in a geriatric hospitalized population. Gerontology. 1992;38 (1-2):111-117. doi:10.1159/000213315

11. Guralnik JM, Eisenstaedt RS, Ferrucci L, et al. Prevalence of anemia in persons 65 years and older in the United States: evidence for a high rate of unexplained anemia. Blood. 2004;104(8):2263-2268. doi:10.1182/blood-2004-05-1812

12. Huang GQ, Lin YT, Wu YM, et al. Individualized prediction of stroke-associated pneumonia for patients with acute ischemic stroke. Clin Interv Aging. 2019;14:1951-1962. doi:10.2147/CIA. S225039

13. Finlayson O, Kapral M, Hall R, et al. Risk factors, inpatient care, and outcomes of pneumonia after ischemic stroke. Neurology. 2011;77 (14):1338-1345. doi:10.1212/WNL.0b013e31823152b1
14. Davydow DS, Hough CL, Levine DA, et al. Functional disability, cognitive impairment, and depression after hospitalization for pneumonia. Am J Med. 2013;126(7):615-24 e5. doi:10.1016/j. amjmed.2012.12.006

15. Lu Y, Liu XY, Chen YJ, et al. Serum iron and A(2)DS(2) score in stroke-associated pneumonia. Int $J$ Clin Exp Med. 2015;8 (4):6163-6170.

16. Garner JS, Jarvis WR, Emori TG, et al. CDC definitions for nosocomial infections, 1988. Am J Infect Control. 1988;16(3):128-140. doi:10.1016/0196-6553(88)90053-3

17. Smith CJ, Kishore AK, Vail A, et al. Diagnosis of stroke-associated pneumonia: recommendations from the pneumonia in stroke consensus group. Stroke. 2015;46(8):2335-2340. doi:10.1161/ STROKEAHA.115.009617

18. Rofes L, Arreola V, Mukherjee R, et al. Sensitivity and specificity of the eating assessment tool and the volume-viscosity swallow test for clinical evaluation of oropharyngeal dysphagia. Neurogastroenterol Motil. 2014;26(9):1256-1265. doi:10.1111/nmo.12382

19. Soares MP, Weiss G. The Iron age of host-microbe interactions. EMBO Rep. 2015;16(11):1482-1500. doi:10.15252/embr.201540558

20. Nairz M, Haschka D, Demetz E, et al. Iron at the interface of immunity and infection. Front Pharmacol. 2014;5:152. doi:10.3389/ fphar.2014.00152

21. Weinberg GA. Iron overload as a mechanism for the lowered survival in AIDS patients receiving dapsone-iron protoxalate for secondary prophylaxis of Pneumocystis carinii pneumonia. J Infect Dis. 1996;174(1):241-242. doi:10.1093/infdis/174.1.241

22. Esan MO, van Hensbroek MB, Nkhoma E, et al. Iron supplementation in HIV-infected Malawian children with anemia: a double-blind, randomized, controlled trial. Clin Infect Dis. 2013;57(11):1626-1634. doi:10.1093/cid/cit528

23. Wang Q, Liu Y, Han L, et al. Risk factors for acute stroke-associated pneumonia and prediction of neutrophil-to-lymphocyte ratios. Am J Emerg Med. 2020;41:55-59. doi:10.1016/j.ajem.2020.12.036
Clinical Interventions in Aging

\section{Publish your work in this journal}

Clinical Interventions in Aging is an international, peer-reviewed journal focusing on evidence-based reports on the value or lack thereof of treatments intended to prevent or delay the onset of maladaptive correlates of aging in human beings. This journal is indexed on PubMed Central, MedLine, CAS, Scopus and the Elsevier
Bibliographic databases. The manuscript management system is completely online and includes a very quick and fair peer-review system, which is all easy to use. Visit http://www.dovepress.com/ testimonials.php to read real quotes from published authors. 